\title{
Modelo Empírico Linear para Previsão de Vazão de Rios na Usina Hidrelétrica de Itaipu - Bacia do Rio Paraná
}

\author{
Maria Gabriela Louzada Malfatti ${ }^{1}$ iD, Andréa de Oliveira Cardoso $^{1}$, Diana Sarita Hamburger ${ }^{1}$ \\ ${ }^{1}$ Centro de Engenharia, Modelagem e Ciências Sociais Aplicadas, \\ Universidade Federal do ABC, Santo André, SP, Brasil.
}

Recebido em 12 de outubro de 2016 - Aceito em 26 de fevereiro de 2018

\begin{abstract}
Resumo
Devido à importância do conhecimento das variações da vazão de rios para o planejamento dos usos múltiplos da água, este estudo objetiva explorar as influências remotas do clima, via padrões de variabilidades climáticas e regionais, via precipitação e vazão em bacias de contribuição. Para tanto, foram desenvolvidos modelos empíricos de previsão de vazões mensais defasados no tempo na Usina Hidrelétrica de Itaipu. Estes modelos foram testados com diferentes grupos de preditores, tais como: índices climáticos; precipitação em regiões pluviométricas homogêneas; vazão em pontos a montante e em Itaipu; e o conjunto de todos os preditores anteriores. Por meio do método stepwise foram selecionados os preditores mais significativos, sendo destacados os seguintes preditores: índices do El Niño Oscilação Sul e de anomalias de temperatura da superfície do mar no Atlântico Tropical Sul; precipitação em locais na faixa sul da bacia; e a própria vazão em Itaipu defasada. Os modelos foram validados, indicando de um modo geral maior desempenho nas defasagens mais curtas, quando considerados os preditores de vazão e precipitação e nas defasagens mais longas, considerando os índices climáticos. Portanto, os resultados deste estudo demonstram a importância de serem consideradas as influências remotas do clima nas estimativas de vazão, principalmente para previsões de longo prazo.
\end{abstract}

Palavras chave: modelo de regressão linear múltipla, previsão de vazão, padrões climáticos.

\section{Linear Empirical Model for Streamflow Forecast in Itaipu Hydroelectric Dam - Parana River Basin}

\begin{abstract}
This study aims to explore the remote climate influences, through patterns of climate variability, and regional, through precipitation and streamflow in contribution of basins, given the meaning of river flow variations knowledge to the planning of water multiple uses. Therefore, empirical models of monthly streamflow in the lower of basin the Parana River at the site Itaipu Dam were developed. These models were tested with different groups of predictors: only the lagged climate indices; only precipitation in homogeneous rainfall regions; only streamflow at upstream points and even the streamflow of Itaipu; and the set of predictors. Through stepwise method the most significant predictors were chosen, being highlighted the best predictors: the indices of the El Niño Southern Oscillation and temperature anomalies of the sea surface in the tropical South Atlantic; rainfall in southern basin sites; and proper lagged streamflow in Itaipu. The models were validated, indicating generally higher performance in shorter lags, when considered predictors of streamflow and precipitation, in the longer lags, it was found that the best performance is for the model considering only the climate indices. Therefore, the results of this study demonstrate the importance of considering the remote climate influences on streamflow estimates, especially for longer-term forecasts.
\end{abstract}

Keywords: multiple linear regression, streamflow forecasting, climate patterns.

Autor de correspondência: Maria Gabriela Louzada Malfatti,mglmalfatti@hotmail.com. 


\section{Introdução}

O estudo da vazão de um curso d'água apresenta várias utilidades uma vez que fornece informações fundamentais sobre o padrão hidrológico de cada rio, úteis para fins de planejamento e tomada de decisão. O comportamento hidrológico de uma bacia é determinado pelas características fisiográficas e climáticas, sendo suas variações influenciadas pelas variabilidades do clima.

Em termos ambientais, além do papel do clima como controlador de variações no sistema hidrológico, este também pode sofrer influências das alterações de superfície da bacia, decorrentes das mudanças de uso e manejo do solo e alteração dos sistemas hídricos. Por exemplo, o desmatamento pode acarretar variações no ciclo hidrológico, influenciando o regime de precipitação, dependendo da extensão da alteração da superfície (D'Almeida et al., 2007). Além disso, o clima local pode ser afetado por construções de barragens, lagos e reservatórios, o que é demostrado pelo estudo de Stivari et al. (2003). Segundo esses autores, a construção da usina de Itaipu causou alteração na circulação local, afetando sistemas de mesoescala como a brisa lacustre, sendo verificado que o contraste térmico associado a presença do lago, induz uma circulação mais forte, atingindo uma extensão horizontal máxima de $50 \mathrm{~km}$, cujo movimento horizontal é afetado pelo efeito de uso do solo.

Devido aos diversos componentes envolvidos que se inter-relacionam e afetam o comportamento hidrológico de uma bacia, é um grande desafio desenvolver estimativas acuradas de vazão. As informações antecipadas das vazões de rios contribuem principalmente para o planejamento operacional de usinas hidrelétricas e de atividades agrícolas, auxiliando na tomada de decisão, que visa minimizar os efeitos de cheias excepcionais e de escassez hídrica (Collischonn et al., 2005).

Vários estudos de previsibilidade de vazão foram desenvolvidos com foco em modelos conceituais que consideram os processos envolvidos na bacia (Collischonn e Tucci, 2001) e até mesmo previsão numérica de precipitação (Guilhon et al., 2007; Cataldi et al., 2007; Bravo et al., 2012).

A consideração da influência das variabilidades climáticas para a estimativa de vazão pode auxiliar no aumento do desempenho dos modelos de previsão de vazão de médio a longo prazo (Souza Filho e Lall, 2004). Este aspecto reforça a influência da precipitação, visando aprimorar e também aumentar o horizonte da previsão acurada e deste modo, o fornecimento de subsídios para o planejamento antecipado.

Neste caso, os modelos empíricos são bastante indicados, pois capturam relações entre variáveis. Estes modelos introduzem as variáveis de entrada e utilizam técnicas matemáticas para ajustar parâmetros empíricos em busca de representar a vazão de saída, sem necessariamente representar o comportamento físico dos processos envolvidos.
As técnicas frequentemente utilizadas são a regressão múltipla, os modelos autoregressivos com média móvel (ARMA, ARIMA) e os modelos não-lineares, como o de redes neurais. Estes modelos geralmente utilizam o conhecimento das estatísticas de séries do passado para estimar as vazões no futuro, portanto dependem da estacionariedade do comportamento da bacia (Tucci, 2005).

A precipitação e, portanto, a vazão sofrem influências remotas de fenômenos climáticos, conforme demonstrado por alguns estudos. Robertson e Mechoso (1998) sugerem que a influência da temperatura da superfície do mar (TSM) sobre as vazões dos rios Uruguai e Paraná ocorre em escala interanual, no caso do Pacífico, e na escala decadal, associada ao Atlântico. Dettinger et al. (2000) mostraram um padrão oposto nas correlações entre vazão de rios sobre as regiões Nordeste e Sul do Brasil e o El Niño-Oscilação Sul (ENOS), no qual a vazão sobre o Nordeste tende a diminuir (aumentar) em anos de El Niño (La Niña), e o contrário foi observado para a região Sul. Cardoso e Silva Dias (2002) analisaram a eficácia dos componentes principais de TSM nos oceanos Atlântico e Pacífico como preditores de vazão em rios do Estado de São Paulo, sendo sugerida a possibilidade de se utilizar padrões de variabilidade de TSM no Pacífico tropical e Atlântico sudoeste para o prognóstico de vazão em rios no Estado de São Paulo.

Berri et al. (2002) identificaram impactos consistentes do ENOS sobre a vazão do Rio Paraná, indicando maiores (menores) fluxos médios nos casos de eventos de El Niño (La Niña) durante o ano de início do fenômeno e até metade do ano seguinte. Grimm e Tedeschi (2009) observaram que o ENOS exerce influência sobre os extremos de precipitação afetando os extremos de vazão em diversas bacias brasileiras. A ocorrência de eventos El Niño (La Niña) está relacionada com o aumento (diminuição) de extremos de vazão na região das Bacias do Paraguai e Uruguai. Kayano e Andreoli (2009) observaram diferenças nos padrões de precipitação associados ao ENOS que podem estar relacionadas à fase da Oscilação Decadal do Pacífico (PDO), dado que as fases do ENOS podem ser influenciadas construtivamente, quando estiverem na mesma fase que a PDO.

Outros estudos apontam que a Oscilação do Atlântico Norte (NAO) (Piccilli, 2007; Siqueira e Molion, 2015), a Oscilação Antártica (AAO) (Oliva, 2011) e a Oscilação Multidecadal do Atlântico (AMO) (Kayano e Capistrano, 2014) podem combinar-se a atuação do ENOS.

O posicionamento e a organização de zonas de convergência podem ser influenciados por padrões climáticos. A Zona de Convergência Intertropical é influenciada pelo Modo Meridional do Atlântico (AMM) (Wainer e Venegas, 2002; Bombardi et al., 2014) e a Zona de Convergência do Atlântico Sul (ZCAS) é influenciada pela AMO (Chiessi et al., 2009) e por anomalias de TSM no Atlântico Tropical Sul (Chaves, 2011). 
Cardoso e Cataldi (2012) destacaram que padrões de teleconexões, oriundos de diferentes padrões climáticos e escalas temporais (de interanual a interdecadal) podem influenciar a vazão de rios brasileiros. Na Bacia do Paraná foram encontradas correlações significativas principalmente com os índices da AAO, AMO, AMM, PDO, NAO, Oscilação Sul (SOI), Atlântico Tropical Sul (TSA) e Anomalias de TSM na região centro leste do Oceano Pacífico Tropical (NIÑO 3.4).

Alguns estudos demonstram a eficiência dos índices climáticos para previsão de vazão em diversas bacias brasileiras. Pinto et al. (2006) utilizam os índices climáticos para previsão de precipitação na bacia do Alto São Francisco, aplicando um modelo de combinação linear ótima de previsões resultantes da autocorrelação serial ou persistência, e dos indicadores climáticos (SOI e anomalias de TSM), que geram uma previsão consensual (as probabilidades de precipitação estão nos limites das categorias). Souza Filho e Lall (2004) utilizaram técnicas de regressão multivariada para explorar a relação entre vazões anuais (ou sazonais) e um conjunto de potenciais preditores climáticos. Ambos os estudos evidenciam a importância da utilização dos índices climáticos e produzem bons resultados.

Alexandre (2012) realizou uma previsão de vazões mensais para o Sistema Integrado Nacional (sistema de produção e transmissão de energia elétrica do Brasil) utilizando informações climáticas, sendo observado que os melhores modelos do tipo Periódico Autorregressivo com variáveis exógenas (PARX) são os que fazem uso de índices climáticos como variáveis exógenas, dentre os quais destacam o índice AMO. Esse índice apresenta melhor desempenho no período seco das bacias do norte do Brasil
(Amazonas e Tocantins-Araguaia) e centro-leste brasileiro (Atlântico Leste e na maioria dos rios que formam a Bacia do Paraná).

Dada a influência de padrões climáticos na vazão, e da importância das variações nos recursos hídricos para as atividades socioeconômicas, é cada vez mais estratégico desenvolver estudos que busquem explorar as influências das variabilidades climáticas. Neste sentido, esta pesquisa objetiva desenvolver um modelo empírico de estimativa de vazões mensais na parte baixa da Bacia do Rio Paraná, mais especificamente no local onde está localizada a Usina Hidrelétrica de Itaipu, a partir de índices de padrões climáticos combinados com a própria vazão defasada, no ponto e em pontos a montante, e com a precipitação em diferentes locais da bacia.

\section{Dados e Metodologia}

\section{1. Área de estudo}

A Bacia do Rio Paraná abrange as bacias hidrográficas do Paraná e Paraguai, inclui parte dos estados de São Paulo, Paraná, Mato Grosso do Sul, Mato Grosso, Minas Gerais, Goiás, Santa Catarina e Distrito Federal, conforme a Fig. 1. Esta região concentra cerca de um terço da população brasileira $(32 \%)$, incluindo a região mais industrializada e urbanizada do Brasil e é a bacia hidrográfica com a maior capacidade instalada de geração de energia elétrica do país, correspondendo a 59,3\% do total nacional, sendo a de maior demanda, cerca de $75 \%$ do consumo nacional (ANA, 2009).

O local da Usina Hidrelétrica de Itaipu foi focalizado para a realização de previsão de vazão. Essa usina possui
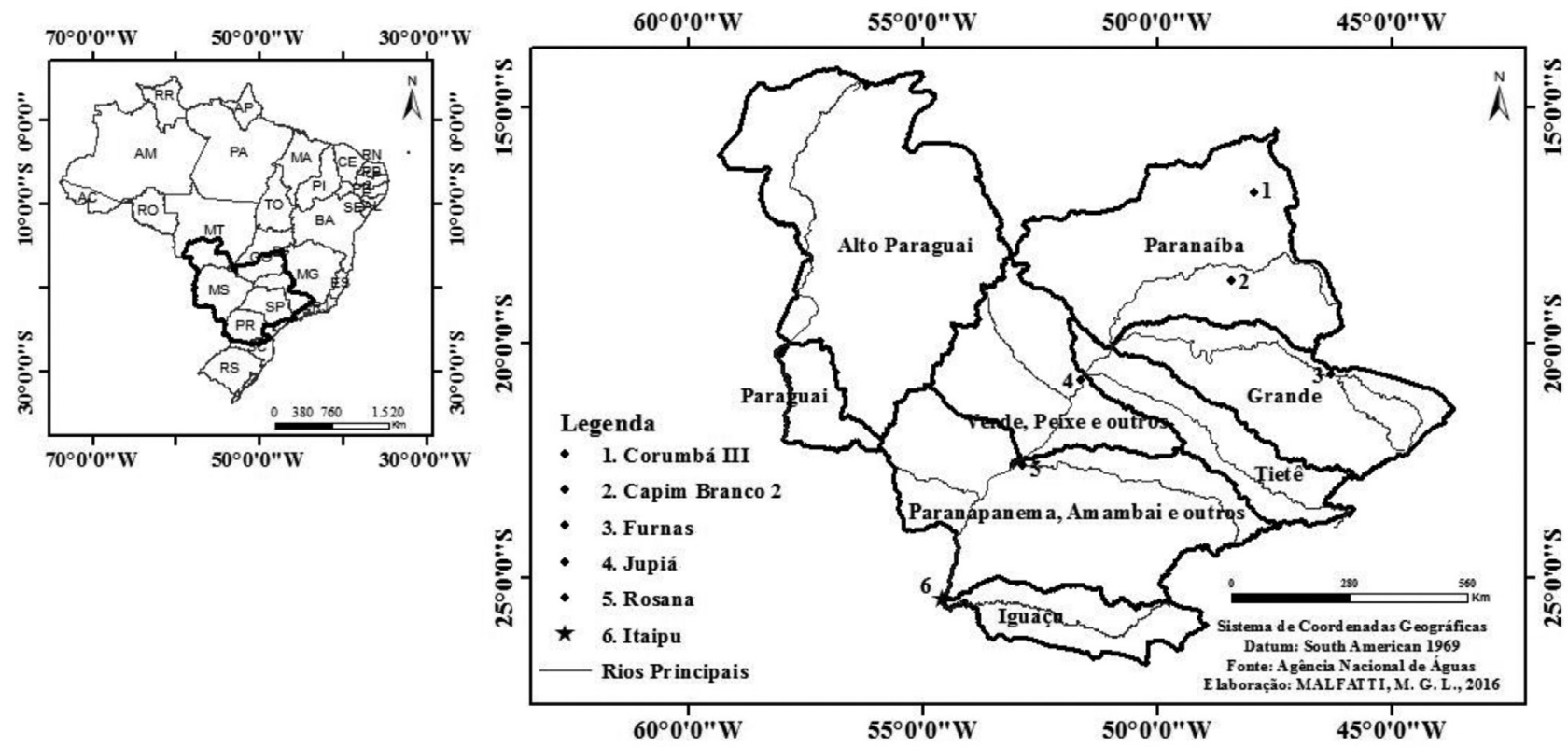

Figura 1 - Localização da Bacia do Rio Paraná, seus principais rios, sub-bacias e as estações fluviométricas utilizadas, com destaque para a Usina Hidrelétrica de Itaipu. 
grande importância energética, estando localizada no Rio Paraná, na fronteira entre o Brasil e o Paraguai. Foi construída no período de 1975 a 1982, Itaipu Binacional, sendo hoje a segunda maior usina do mundo em potencial instalado e a primeira em total de geração. Sua maior produção foi no ano de 2016, atingindo 103,09 milhões MWh, fornecendo cerca de $15 \%$ da energia consumida no Brasil e $86 \%$ do consumo paraguaio (ANA, 2009; Itaipu Binacional, 2018).

\subsection{Dados}

Para o desenvolvimento deste estudo foram utilizados dados mensais de índices climáticos, vazões de rios e precipitação no período de 1980 a 2010 .

Os dados mensais de índices climáticos utilizados estão disponíveis no site do National Oceanic and Atmospheric Administration (NOAA), sendo utilizados os índices: Oscilação Antártica (AAO); Modo Meridional do Atlântico (AMM); Oscilação Multidecadal do Atlântico (AMO); Oscilação do Atlântico Norte (NAO); Anomalias de TSM na região centro-leste do Oceano Pacífico Tropical (NIÑO 3.4); Oscilação Decadal do Pacífico (PDO); Índice Oscilação Sul (SOI) e Índice do Atlântico Tropical Sul (TSA).

Os dados de vazões utilizados estão disponíveis no site do Operador Nacional do Sistema Elétrico (ONS). O conjunto de dados do ONS é composto por informações de vazões naturais afluentes em aproveitamentos hidroelétricos no Brasil. O termo vazão natural tem sido adotado pelo setor elétrico para identificar a vazão que ocorreria em uma seção do rio, se não houvesse as ações antrópicas na sua bacia contribuinte, tais como regularização de vazões realizadas por reservatórios, desvios de água, evaporações em reservatórios e usos consuntivos (irrigação, criação animal e abastecimento urbano, rural e industrial). Obtida por meio de um processo de reconstituição, que considera a vazão observada no local e as informações relativas às ações antrópicas na bacia (Cataldi et al., 2007). Neste trabalho, foram utilizadas as vazões naturais mensais distribuídas em pontos estratégicos sobre a Bacia do Rio Paraná, sendo selecionados pontos de sub-bacias a montante que apresentaram maior correlação com Itaipu.

Para os dados de precipitação mensais foram utilizadas séries histórias médias em regiões pluviométricas homogêneas definidas em Malfatti (2016), gerados a partir de dados disponíveis na Agência Nacional das Águas (ANA). Neste estudo foram encontradas oito regiões com comportamentos distintos na bacia do Rio Paraná entre os anos de 1980 a 2010, destacadas na Fig. 2.

Ambos os conjuntos de dados foram padronizados, permitindo que as séries diferentes pudessem ser representadas em escalas comparáveis. Para o caso da precipitação, foi realizada a padronização mensal, pela média e desvio padrão de cada mês correspondente, destacando as variabilidades e, removendo a sazonalidade, conforme a Eq. (1).

$$
x^{\prime}(i, j)=\frac{x(i, j)-\mu(j)}{S(j)}
$$

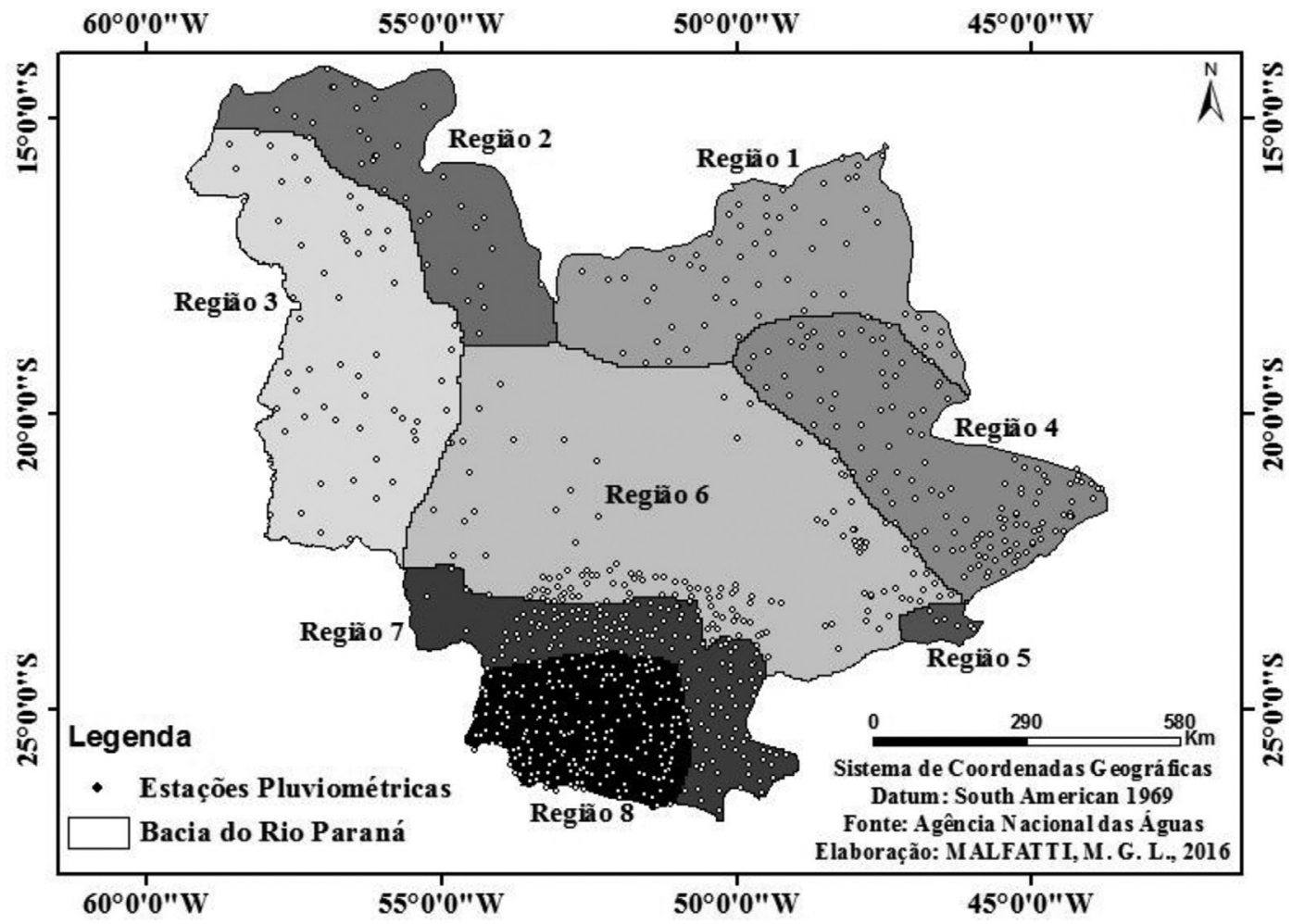

Figura 2 - Regiões pluviométricas homogêneas na bacia do Rio Paraná (Malfatti, 2016). 
sendo que $i$ é o índice do ano e $j$ o índice do mês; é o valor padronizado da variável do j-ésimo mês do i-ésimo ano, e onde é a média para um mês $j$ e pode também ser chamada de ciclo sazonal; é o desvio padrão do mês $j$.

No caso dos índices climáticos a padronização foi realizada sobre toda a série de dados.

\subsection{Modelo empírico de regressão linear múltipla}

O Modelo de Regressão Linear Múltipla é um modelo empírico que, ao ser construído considera as relações empíricas entre as variáveis observadas, sem necessariamente representar o comportamento físico dos processos, sendo de fácil ajuste e validação. Este modelo é aplicado para uma variável que se deseja estimar, cuja série histórica apresenta fortes relações com outras variáreis, sendo que essas relações se associam ao fenômeno (ou processo) que se deseja modelar. Como este estudo busca analisar as melhores variáveis que tornam o modelo mais preciso, não considerando somente as próprias vazões defasadas, mas também outras variáveis em diferentes defasagens, como a precipitação e os índices climáticos, tal método atende o objetivo do estudo, assim como em outros estudos que demonstram sua habilidade para tais fins (Cardoso e Silva Dias, 2002; Grimm et al., 2004).

Cardoso (2005) utilizou este modelo para verificar a relação entre a TSM nos oceanos Atlântico e Pacífico e as condições climáticas nas regiões Sul e Sudeste do Brasil. Alexandre (2012) utilizou este modelo para analisar o impacto da incorporação de informações climáticas na previsão de vazões mensais para o Sistema Interligado Nacional. Malfatti et al. (2014) utilizaram este modelo para previsão de vazão na usina hidrelétrica de Itaipu, a partir de séries de vazão e precipitação.

O modelo de Regressão Linear Múltipla fornece a relação entre uma determinada variável dependente e outras variáveis independentes, pelo método dos mínimos quadrados (Wilks, 2006). A equação de regressão (Eq. (2)) possui a seguinte forma:

$$
y=b_{0}+b_{1} X_{1}+b_{2} X_{2}+b_{3} X_{3}+\cdots+b_{k} X_{k}
$$

sendo $y$ a variável dependente (vazão a ser prevista); $X_{n}$ são as variáveis independentes (variáveis escolhidas que contribuem para a variação de vazão); $b_{0}$ é a intersecção da reta com o eixo de y; $b_{n}$ são os coeficientes angulares obtidos da regressão; $k$ é o número de variáveis independentes.

Neste trabalho foram desenvolvidos modelos mensais utilizando: $i$ ) modelos com apenas índices climáticos; ii) modelos com apenas precipitação nas regiões homogêneas; iii) modelos com apenas vazões a montante e Itaipu defasada e $i v$ ) modelos com todos os itens anteriores.

\subsection{Seleção dos preditores - Método stepwise}

No conjunto de variáveis independentes pode haver variáveis que apresentam pouca influencia na variável dependente, não contribuindo para a melhoria da estimativa ao se agregar ao conjunto de preditores. O método stepwise é utilizado para adicionar e remover variáveis independentes de um modelo de regressão linear múltipla com base em sua significância estatística, podendo assim, reduzir o número de variáveis que compõe a equação de regressão (Draper e Smith, 1998). Este método é realizado de forma interativa, adicionando e removendo variáveis, a partir de um critério de seleção, sendo o teste $\mathrm{F}$ o critério utilizado para a escolha das variáveis que contribuem significativamente para a regressão (Draper e Smith, 1998).

No presente estudo, o método stepwise foi aplicado para selecionar as variáveis de precipitações, índices climáticos e vazões, separadamente, visando a configuração de modelos mais robustos.

\subsection{Calibração e validação do modelo}

$\mathrm{Na}$ validação do modelo busca-se atingir o melhor ajuste, ou seja, nesta etapa determina-se o grau em que o modelo é capaz de realizar uma representação precisa do mundo real a partir da perspectiva de suas simulações.

Para o desenvolvimento e avaliação do modelo, divide-se o conjunto de dados em dois períodos, o primeiro é utilizado na calibração, para o ajuste da equação de regressão, e o segundo período é considerado no processo de validação, comparando o conjunto de pares previsão-observação, cuja observação não foi incluída na calibração. Tal método é usualmente aplicado no desenvolvimento de modelagem empírica, visando obter o melhor arranjo empírico e desempenho do modelo através dos preditores considerados (Krauskopf Neto et al., 2007; Ratton e Guetter, 2015; Câmara et al., 2016).

O período de dados utilizado na etapa de calibração dos modelos foi de 1980 a 2000, considerando cada mês, separadamente. Neste processo foi calculado o coeficiente de determinação $\mathrm{R}^{2}$, que é uma medida do poder explicativo do modelo de regressão, o qual fornece a proporção da variação da variável dependente, que é explicada em termos lineares pelas variáveis independentes (Wilks, 2006). O R é calculado pela Eq. (3).

$$
R^{2}=\frac{\frac{n \sum_{j=1}^{n} y_{i c}^{2}-\left(\sum_{j=1}^{n} y_{i}\right)^{2}}{n(n-1)}}{\frac{n \sum_{j=1}^{n} y_{i}^{2}-\left(\sum_{j=1}^{n} y_{i}\right)^{2}}{n(n-1)}}
$$

onde $y_{i c}$ é o valor calculado através da função de regressão e $y_{i}$ é o valor observado, $n$ é o número de observações consideradas na calibração.

$\mathrm{O}$ coeficiente $\mathrm{R}^{2}$ é obtido através da análise de variância, na qual se determina o quanto da variabilidade da variável dependente é considerado pela regressão. Os valores de $\mathrm{R}^{2}$ podem variar entre 0 e 1 , quando este valor for 1 a regressão foi perfeita, sendo que, o inverso ocorre quando o valor de $\mathrm{R}^{2}$ se aproxima de 0 (zero) (Wilks, 2006). 
Para validar o modelo é necessário avaliar o quanto ele é capaz de reproduzir a realidade, aplicando uma avaliação nas saídas do modelo, baseado em um período não considerado na calibração. Esta avaliação consiste na identificação de possíveis limitações do modelo, que possam exigir um novo processo de ajuste e validação, que no caso de modelos empíricos poderia ser o aumento da série histórica, incluindo períodos mais recentes de observação, como também a inclusão de outros preditores. Neste estudo, os dados utilizados para esta etapa de validação englobam anos de 2001 a 2010.

Neste processo foram calculados os seguintes parâmetros para a avaliação do desempenho do modelo: Coeficiente de Correlação (CC), Erro Viés (VIES), Raiz do Erro Quadrático Médio (REQM), Erro Absoluto (EA) e o Erro Relativo (ER) (Wilks, 2006) e o Coeficiente de NashSutcliffe (CN) (Collischonn, 2010), conforme apresentado nas Eqs. (4) a (9).

$$
C C=\frac{\sum_{i=1}^{n} x_{i} y_{i}-\frac{1}{n}\left(\sum_{i=1}^{n} x_{i}\right)\left(\sum_{i=1}^{n} y_{i}\right)}{\sqrt{\sum_{i=1}^{n} x_{i}^{2}-\frac{1}{n}\left(\sum_{i=1}^{n} x_{i}\right)^{2}} \sqrt{\sum_{i=1}^{n} y_{i}^{2}-\frac{1}{n}\left(\sum_{i=1}^{n} y_{i}\right)^{2}}}
$$

sendo $x_{i}$ e $y_{i}$ são as variáveis padronizadas a serem correlacionadas.

$$
\begin{aligned}
& \text { VIES }=\frac{1}{n} \sum(F-O) \\
& R E Q M=\sqrt{\frac{1}{n} \sum(F-O)^{2}} \\
& E A=\frac{1}{n} \sum|F-O| \\
& E R=\frac{1}{n} \sum \frac{|F-O|}{O} \\
& C N=1-\frac{\sum_{i=1}^{n}(O-F)^{2}}{\sum_{i=1}^{n}(O-\bar{O})^{2}}
\end{aligned}
$$

sendo $F$ o valor estimado pelo modelo, $O$ o valor observado e $\bar{O}$ o valor médio observado.

\section{Resultados e Discussão}

A regressão por stepwise conduzida para cada mês separadamente, para fins de estimativa de vazão, permitiu a identificação de grupos de preditores variados ao longo do ano e das defasagens testadas.

Os resultados da seleção de preditores pelo método de stepwise apontam que os índices NIÑO 3.4 e SOI aparecem como preditores na maioria dos casos, influenciando a vazão de Itaipu nos meses de abril a novembro em diferentes defasagens. Esses dois índices evidenciam a influência do ENOS sobre a vazão em Itaipu ao longo do ano, que pode ser explorada para fins de previsão, com exceção do verão (dezembro a fevereiro) e início do outono (março).

$\mathrm{O}$ índice TSA foi apontado como preditor nas defasagens mais longas para os meses abril, novembro e dezembro, e nas primeiras defasagens para o mês de julho, enquanto que o índice $\mathrm{AMO}$ foi selecionado em quase todos os meses, exceto julho, setembro e dezembro, em diferentes defasagens (lag 4 a 12). Para o índice AAO o método sugere os meses de janeiro, março, abril, maio, agosto, novembro e dezembro como preditor, normalmente nas defasagens mais longas (a partir do lag 5).

Nota-se também que os índices AMM, NAO e PDO foram os índices selecionados com menor frequência ao longo dos meses, o índice AMM é indicado para os meses de abril, novembro e dezembro, destacando o mês de novembro com maior número de defasagens (lag 5 a lag 8 e lag 10), o índice NAO apenas para os meses de abril (lag 8), agosto (lag 5), setembro (lag 6 e 12) e outubro (lag 12). E, por fim, o índice PDO aparece apenas como preditor significativo nos meses de setembro e dezembro, para o mês de setembro em defasagem mais longa (lag 8), enquanto que para o mês de dezembro com um mês de defasagem.

Na seleção de preditores de precipitação, as regiões pluviométricas homogêneas 8 (sul da bacia) e 6 (faixa central da bacia) se destacam, sendo que a região 8 foi selecionada pelo método para todos os meses do ano em diferentes defasagens e a região 6 só não foi selecionada para os meses de setembro e outubro, sendo esta última a região que aparece mais vezes como preditora na defasagem de um mês, indicando que a contribuição da precipitação nesta região demora no máximo um mês para contribuir para a vazão em Itaipu.

Nota-se que a região 7 (sul da bacia) influencia no segundo semestre do ano, principalmente no período de inverno, sugerindo que a vazão nesta época do ano tem contribuição maior da precipitação derivada da faixa sul da bacia do Paraná, região com um padrão de chuva mais regular ao longo do ano, dado que esse é o período seco na parte alta da bacia. Em contrapartida, a região 1 (nordeste da bacia) influencia mais no primeiro semestre, em especial nos meses de abril e maio, e a região 2 (norte da bacia) influencia no outono, com defasagens mais longas, e na primavera, com defasagens mais curtas.

As regiões 3, 4 e 5 (oeste e leste da bacia) são as regiões que foram selecionadas pelo método com menor frequência, destacando a região 3 , nos meses de abril e setembro, a região 4, em maio, junho e dezembro e a região 5 em março e junho.

Para o caso das vazões em pontos a montante, observou-se que, no geral, para as defasagens mais longas não foram selecionados preditores de vazão pelo método stepwise, com exceção dos meses abril, outubro, novembro e dezembro, que indicam alguns postos nas defasagens de nove a doze meses. Em abril, o posto mais distante Corumbá III aparece na defasagem 10, sugerindo uma contri- 
buição do escoamento de base desse posto para a vazão de Itaipu, enquanto que nos meses de outubro, novembro e dezembro, os postos mais próximos de Itaipu foram selecionados pelo método.

Para quantificar a contribuição de cada grupo de preditores selecionados foram calibrados e validados os modelos desenvolvidos para as doze defasagens em todos os meses, e para os seguintes grupos de preditores: somente os índices climáticos; somente precipitação em regiões homogêneas; somente vazões em ponto a montante e vazão em Itaipu defasada; e o conjunto de todos os preditores anteriores. Foram considerados os preditores selecionados para cada defasagem, ou seja, os modelos foram construídos conforme os preditores selecionados pelo método stepwise para cada defasagem e para cada mês correspondente. No caso em que nenhum preditor foi apontado como significativo pelo método, considerou-se apenas a própria vazão de Itaipu defasada.

Os resultados da calibração, através do coeficiente de determinação $\left(\mathrm{R}^{2}\right)$, para o modelo considerando todos os grupos de preditores (índices, precipitação e vazão) para cada defasagem (1-12 meses) de janeiro a dezembro no período de 1980 a 2000, e na validação para o período de 2001 a 2010, através dos parâmetros VIES, REQM, EA e ER estão apresentados na Fig. 3.

A partir dos valores apresentados de $\mathrm{R}^{2}$ (coeficiente de determinação) pode-se inferir sobre a calibração dos modelos, considerando cada grupo de preditores separadamente (os índices, a precipitação e a vazão), sendo verificado que para os meses janeiro, fevereiro e março os índices climáticos não contribuem para a previsão de vazão nas primeiras defasagens (lag 1 a 3), a contribuição dos índices será importante nas defasagens mais longas lag 6, lag 5 e lag 4 para os meses de janeiro, fevereiro e março respectivamente. Para os meses de abril e maio a contribuição dos índices climáticos é evidenciada desde as primeiras defasagens, para o mês de maio o $\mathrm{R}^{2}$ atinge o valor de 0,76 para o lag 3 . No mês de junho e julho os valores de $\mathrm{R}^{2}$ para os índices climáticos continuam altos, mas sua contribuição é menor para as defasagens mais curtas. Nos meses seguintes, a contribuição dos índices é novamente comprovada nas defasagens mais longas, sendo que nas defasagens acima de oito meses, apenas os índices climáticos conseguem obter preditores significativos, sendo os únicos preditores de vazão de Itaipu nas defasagens mais longas. Em contrapartida, quando são considerados todos os grupos de preditores (Fig. 3) observa-se que os valores de $\mathrm{R}^{2}$ no geral são maiores para defasagens mais curtas, porém alguns meses apresentam boa calibração em todas as defasagens analisadas, destaque para os meses de outubro e novembro.

Conforme a validação no período de 2001-2010, considerando todos os grupos de preditores, os modelos tendem a superestimar os valores observados, evidenciados pelos altos valores positivos de viés, com exceção de alguns casos, por exemplo no mês de dezembro, na maioria das defasagens os modelos subestimam os valores observados (valores negativos de viés). Além disso, os meses de fevereiro e outubro apresentam menores erros sistemáticos. Nota-se também que alguns meses que apresentaram altos valores $\mathrm{R}^{2}$ (março a junho) possuem o viés mais alto, associados a superestimativa.

Avaliando os resultados para de REQM, todos os meses apresentam valores similares, em torno de $1.000 \mathrm{a}$ $1.500 \mathrm{~m}^{3} / \mathrm{s}$, com execeção do mês de janeiro que apreseta valor discrepante de $2.000 \mathrm{~m}^{3} / \mathrm{s}$ no lag 6 e para o mês de agosto qua apresenta valores entre 400 a $600 \mathrm{~m}^{3} / \mathrm{s}$. Para o EA o mês de janeiro também apresenta os maiores valores em todas as defasagens analisadas, sendo o mês de maior incerteza da previsão. Enquanto que, de acordo com o ER os meses de setembro e outubro apresentam os valores mais elevados na maioria das defasagens.

Para alguns meses e defasagens específicas observase que o modelo apresenta uma boa calibração, expressos pelo altos valores de $\mathrm{R}^{2}$, porém em sua validação apresentam erros altos, evidenciando a aleatoriedade do processo, ou seja, o modelo escolhido captura apenas as relações dos processo no período de ajuste. Deste modo, nesses casos é necessário avaliar outros modelos empíricos que possam capturar as relações não lineares, como é o caso de modelos de inteligencia artificial (Bravo, 2010; Oliveira et al., 2015; Araújo et al., 2015).

Os gráficos apresentados na Fig. 4 comparam as séries estimadas pelos modelos no período de validação com a série observada, para as doze defasagens analisadas, incluindo os respectivos coeficientes de correlação (CC) e o coeficiente de Nash-Sutcliffe $(\mathrm{CN})$. A partir desta figura observa-se que nas primeiras defasagens, os modelos conseguem captar as variabilidades e os extremos, com destaque para o lag 1. Nas defasagens de 4 a 6 meses as variabilidades são capturadas, no entanto os extremos são muitas vezes superestimados ou subestimados, o que é observado com maior frequencia nas defasagens seguintes de 7 a 12 meses. Em contrapartida, alguns picos são estimados nas defasagens mais longas, ao se considerar apenas preditores de índices climáticos, demostrando a importância de serem consideradas as influências remotas do clima nas estimativas de vazão, principalmente para previsões de mais longo prazo. Observa-se também que o coeficiente de Nash-Stucliffe apresenta valores entre 0,75 e 0,36 (desemprenho aceitável, de acordo com Collischonn, 2010) em quase todas as defasagens, com exceção do lag 6, que não apresenta um desempenho aceitável $(\mathrm{CN}=0,34)$. $\mathrm{Na}$ análise do coeficiente de correlação os resultados também são aceitáveis, acima de 0,70 para quase todas as defasagens, destacando mais uma vez o lag 6 que não apresenta resultados satisfatórios, com coeficiente de correlação igual a 0,67 .

No ano de 2007 observa-se uma grande anomalia positiva não capturada pelos modelos, principalmente com 
defasagens mais longas, sendo apenas atingida uma boa estimativa com um mês de antecedência. Segundo o Boletim de Informações Climáticas (INFOCLIMA) elaboradas pelo grupo de Clima do CPETC/INPE, as características atmosféricas observadas no início do ano mostraram um verão atípico, com alagamentos, enxurradas e des- lizamentos, foram observados principalmente sobre as regiões Sudeste e Centro-Oeste. Nestas áreas, a ocorrência de chuvas intensas foi decorrente da atuação de sistemas frontais semi-estacionários, contribuindo para a configuração de um episódio de ZCAS durante quase todo o mês de janeiro. Os totais observados estiveram muito acima da mé-
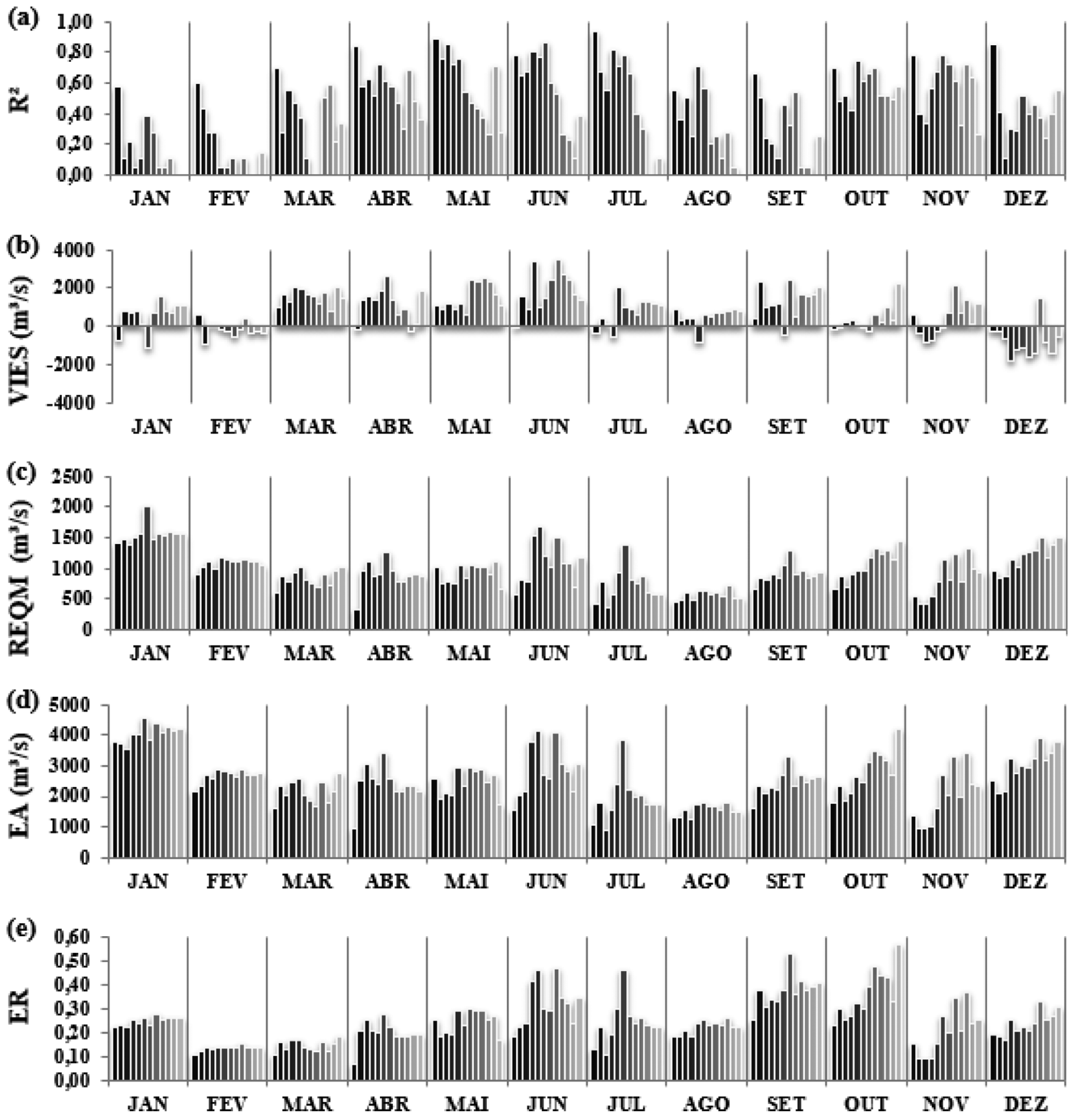

\section{Legenda:}

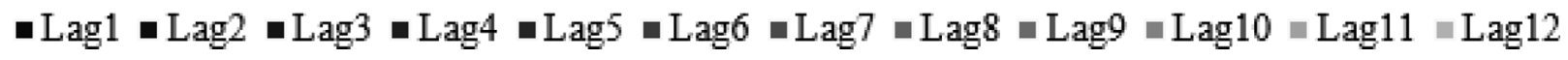

Figura 3 - Resultados da validação do modelo para previsão de vazão mensal considerando todos os grupos de preditores (índice, precipitação e vazão) defasados em doze meses, onde: a) coeficiente de determinação $\left(\mathrm{R}^{2}\right)$; b) erro viés (VIES); c) raiz do erro quadrático médio (REQM); d) erro absoluto (EA); e e) erro relativo (ER). 

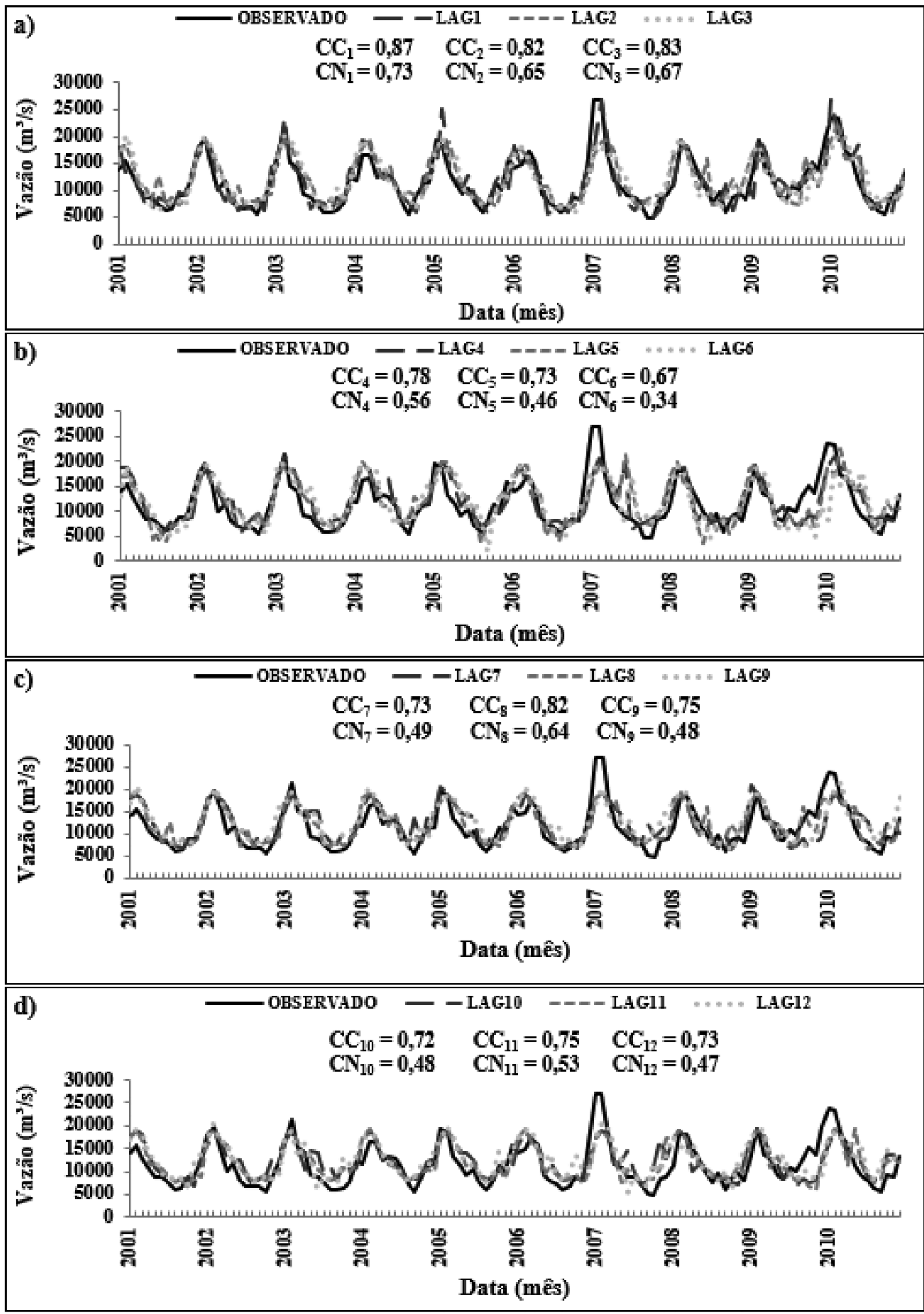

Figura 4 - Comparação do modelo com o observado para as doze defasagens e os respectivos coeficientes de correlação (CC) e coeficiente de Nash-Sutcliffe (CN), onde: a) observado e estimado nas defasagens 1 a 3 ; b) observado e estimado nas defasagens 4 a 6 ; c) observado e estimado nas defasagens 7 a 9 ; e d) observado e estimado nas defasagens 10 a 12 . 
dia histórica (aproximadamente $80 \%$ ), resultando em um caso atípico, estando entre os cinco janeiros mais chuvosos dos últimos 46 anos. Este episódio resultou em um grande escoamento superficial nos afluentes do Rio Paraná, conduzindo a um aumento expressivo na vazão em Itaipu.

Para o ano de 2010 observa-se outra anomalia positiva não capturada pelos modelos, também nas defasagens mais longas, sendo apenas atingida uma boa estimativa com um mês de antecedência, situação similar encontrada ao ano de 2007. Segundo o INFOCLIMA o excesso de chuva no centro-sul do Brasil esteve associado principalmente à atuação de vórtices ciclônicos em altos níveis sobre a região Nordeste do Brasil e ao escoamento da corrente de jato em baixos níveis trazendo umidade da região tropical para as Regiões Sul e Sudeste durante os meses de janeiro e início de fevereiro.

\section{Conclusões}

Este trabalho permitiu a identificação de diferentes preditores de vazão em Itaipu, em meses e defasagens específicas. Em relação aos índices climáticos, destacaram-se entre janeiro e março apenas os índices AMO e AAO, em abril e julho, os índices NIÑO 3.4 e TSA, em maio, junho, agosto e outubro, NIÑO 3.4 e SOI, em setembro o NAO, em novembro, TSA e AMM, e por fim em dezembro, somente o índice TSA. Em suma, os resultados sugerem que a vazão de Itaipu é influenciada principalmente pelo ENOS (índices NIÑO 3.4 e SOI) ao longo do ano, exceto no verão (dezembro, janeiro e fevereiro) e pelas anomalias de TSM no Atlântico tropical sul (índice TSA), no final do outono e da primavera e no início do inverno e do verão.

Para os preditores de precipitação as regiões que mais foram apontadas pela métrica aplicada estão localizadas ao sul, norte e no centro da Bacia do Paraná. Para o caso das vazões, observou-se que no geral, a vazão da própria Itaipu defasada é indicada como preditora com maior frequência ao longo dos meses, principalmente nas defasagens mais curtas.

De um modo geral, os modelos mensais apresentaram um maior desempenho nas defasagens mais curtas, quando considerados os preditores de vazão e precipitação, exceto para os meses de janeiro, julho e agosto, em que a precipitação tem maior contribuição. Nestes casos são bem capturadas as variabilidades e alguns extremos. Em contrapartida, para o mês de maio, todos os modelos possuem um baixo desempenho, não sendo capaz de estimar as variabilidades e os extremos. Para todos os meses, verificou-se que o melhor desempenho para as defasagens mais longas ocorre para o modelo considerando somente os índices climáticos, mas na maioria dos casos os extremos não são capturados, exceto nos meses de abril, junho e julho.

Os resultados deste estudo demonstram a importância de serem consideradas as influências remotas do clima nas estimativas de vazão, com abordagem mensal, pois os impactos dos padrões climáticos são diferenciados ao longo do ano, e apresentam influências significativas com defasagens distintas, necessitando serem explorados de forma específica. Também sugere que a consideração de padrões de variabilidades climáticas de mais alta frequência pode vir a contribuir para um aumento do desempenho do modelo, como por exemplo, as variabilidades intrasazonais, pois alguns casos extremos não detectados foram decorrentes de fenômenos nesta escala de tempo. Neste sentido, os modelos empíricos são bastante úteis, pois podem facilmente ser ajustados a diferentes localidades, períodos e conjuntos de preditores.

\section{Agradecimentos}

Os autores agradecem à UFABC pelo apoio técnico e financeiro, à CAPES pela bolsa de mestrado do primeiro autor e ao $\mathrm{CNPq}$, pelo apoio ao Projeto Universal $\left(\mathrm{n}^{\circ}\right.$ 471700/2013-4).

\section{Referências}

ALEXANDRE, A.M.B. Previsão de vazões mensais para o sistema interligado nacional utilizando informações climáticas. 2012. 293 f. Tese (Doutorado em Engenharia Civil) - Departamento de Engenharia Hidráulica e Ambiental, Universidade Federal do Ceará, Fortaleza, 2012.

ANA. Agência Nacional de Águas (Brasil). Relatório de Conjuntura dos Recursos Hídricos do Brasil. Brasília, 2009. $204 \mathrm{p}$.

ARAÚJO, C.B.C.; DANTAS NETO, S.A.; SOUZA FILHO, F. de A. Previsão de vazões para o açude Orós/CE a partir de dados hidrometeorológicos utilizando perceptrons. Revista Brasileira de Meteorologia, v. 30, n. 1, p. 37-46, 2005.

BERRI, G.J.; GHIETTO, M.A.; GARCIA, N.O. The influence of ENSO in the flows of the upper Paraná River of South America over the past 100 years.Journal of Hydrometeorology, v. 3, n. 1,p. 57-65, 2002.

BOMBARDI, R.J.; CARVALHO, L.M.V.; JONES, C.; REBOITA, M.S. Precipitation over eastern South America and the South Atlantic sea surface temperature during neutral ENSO periods. Climate Dynamics, v. 42, n. 6, p. 1553-1568, 2014.

BRAVO, J.M. Subsídios à operação de reservatórios baseada na previsão de variáveis hidrológica. 2010. 205 f. Tese (Doutorado em Engenharia de Recursos Hídricos e Saneamento Ambiental) - Instituto de Pesquisas Hidráulicas, Universidade Federal do Rio Grande do Sul, Porto Alegre, 2010.

BRAVO, J.M.; ALLASIA, D.; PAZ, A.R.; COLLISCHONN, W.; TUCCI, C.E.M. Coupled hydrologic-hydraulic modeling of the upper Paraguay River Basin. Journal of Hydrologic Engineering, v. 17, n. 5, p. 635-646, 2012.

CÂMARA，R.K.C.; ROCHA， E.J.P.; PROTÁZIO， J.M.B.; QUEIROZ, J.C.; RIBEIRO, W.M.N. et al. Hydrological stochastic modeling applied to the Tocantins River to the city of Maraba (PA). Revista Brasileira de Meteorologia, v. 31, n. 1, p. 11-23, 2016.

CARDOSO, A.O.; SILVA DIAS, P.L. Relação entre a vazão de alguns rios do Estado de São Paulo e anomalias de TSM no Atlântico e Pacífico. In: Congresso Brasileiro de Meteo- 
rologia, 12., 2002, Foz do Iguaçu. Anais... Rio de Janeiro: SBMET, 2002.

CARDOSO, A.O. Relações entre a TSM nos oceanos Atlântico e Pacífico e as condições climáticas nas regiões Sul e Sudeste do Brasil. 2005. 192 f. Tese (Doutorado de Meteorologia) - Instituto de Astronomia Geofísica e Ciências Atmosféricas, Universidade São Paulo, São Paulo, 2005.

CARDOSO, A.O.; CATALDI,M.Relações de índices climáticos e vazão de rios no Brasil.In:Congresso Brasileiro de Meteorologia, 17., 2012, Gramado. Anais... Rio de Janeiro: SBMET, 2012.

CATALDI, M.; MACHADO, C.O.; GUILHON, L.G.F.; CHOU, S.C.; GOMES, J.L. et al. Análise das previsões de precipitação obtidas com a utilização do Modelo ETA como Insumo para modelos de previsão semanal de vazão natural. Revista Brasileira de Recursos Hídricos, v. 12, n. 3, p. 5-12, 2007.

CHAVES, R. R. Conexões entre a TSM do Atlântico Sul e a Convecção de verão sobre a América do Sul - Análise observacional. Revista Brasileira de Geofísica, v. 29, n. 1, p. 5-10, 2011.

CHIESSI, C.M.; MULITZA, S.; PäTZOLD, J.; WEFER G.; MARENGO, J.A. Possible impact of the Atlantic Multidecadal Oscillation on the South American summer monsoon. Geophysical Research Letters, v. 36, n. 21, p. 1-5, 2009.

COLLISCHONN, W.; TUCCI, C.E.M. Simulação hidrológica de grandes bacias. Revista Brasileira de Recursos Hídricos, v. 6, n. 1, p. 95-118, 2001.

COLLISCHONN, W.; TUCCI, C.E.M.; CLARKE, R.T.; SILVA DIAS, P.L.; OLIVEIRA, G.S. Previsão sazonal de vazão na Bacia do Rio Uruguai 2: Previsão climática-hidrológica. Revista Brasileira de Recursos Hídricos, v. 10, n. 4, p. 61-72, 2005.

COLLISCHONN, W.; Simulação hidrológica de grandes bacias. 2010. 194 f. Tese (Doutorado em Engenharia de Recursos Hídricos e Saneamento Ambiental) - Instituto de Pesquisas Hidráulicas, Universidade Federal do Rio Grande do Sul, Porto Alegre, 2010.

D'ALMEIDA, C.; VÖRÖSMARTY, C.J.; HURTT, G.C.; MARENGO, J.A.; DINGMAN, S.L. et al. The effects of deforestation on the hydrological cycle in Amazonia: a review on scale and resolution. International Journal of Climatology, v. 27, n. 5, p. 633-647, 2007.

DRAPER, N.R.; SMITH, H. Applied regression analysis. 3 ed. Nova Jersey: Wiley, 1998. 706p.

DETTINGER, M.D.; CAYAN, D.R.; MCCABE, G.J.; MARENGO, J.A. Multiscale streamflow variability associated with El Nino/Southern Oscillation. In: DIAZ, H. F.; MARKGRAF, V. (Org.). EI Nino and the Southern Oscillation Multiscale Variability, Global and Regional Impacts, Cambridge: University Press, 2000. P. 114-147.

GRIMM, A.M.; LEITE, A.E.; SAHAIR, A.K. Previsão de precipitação sazonal para a Bacia do Rio Paranaíba utilizando um modelo linear. In: Congresso Brasileiro de Meteorologia, 13., 2004, Fortaleza. Anais... Rio de Janeiro: SBMET, 2004.

GRIMM, A.M.; TEDESCHI, R.G. ENSO and extreme rainfall events in South America. Journal of Climate, v. 22, n. 7, p. 1589-1609, 2009.

GUILHON, L.G.F.; ROCHA, V.F.; MOREIRA. J.C. Comparação de métodos de previsão de vazões naturais afluentes a aproveitamentos hidroelétricos. Revista Brasileira de Recursos Hídricos, v. 12, n. 3, p. 13-20, 2007.

ITAIPU BINACIONAL (ITAIPU). Disponível em: http://www.itaipu.gov.br/. Acesso: 10/05/2018.

KAYANO, M.T.; ANDREOLI, R.V. Variabilidade decenal a multidecenal. In: Cavalcanti, I.F.A.; FERREIRA, N.J.; SILVA, M.G.A.J.; SILVA DIAS, M.A.F. Tempo e Clima no Brasil. 1 ed. São Paulo: Oficina de Textos, 2009. p. $375-383$.

KAYANO, M.T.; CAPISTRANO, V.B. How the Atlantic multidecadal oscillation (AMO) modifies the ENSO influence on the South American rainfall. International Journal of Climatology, v. 34, n. 1, p. 162-178, 2014.

KRAUSKOPF NETO, R.; GUETTER, A.K.; MINE, M.R.M. Modelo hidrológico com atualização de estado Parte II: Estimador de Estado Dinâmico-Estocástico. Revista Brasileira de Recursos Hídricos, v. 12, n. 3, p. 105-115, 2007.

MALFATTI, M.G.L.; LINGIARDI, A.M.; CARDOSO, A.O. Modelo Empírico para previsão de vazão de rios - Caso Itaipu. In: Congresso Brasileiro de Meteorologia, 18., 2014, Recife, Anais Rio de Janeiro: SBMET, 2014.

MALFATTI, M.G.L. Estudo e desenvolvimento de modelagem para previsão de vazão de rios na Bacia do Paraná. 2016. 155 f. Dissertação (Mestrado em Ciência e Tecnologia Ambiental) - Centro de Engenharia, Modelagem e Ciências Sociais Aplicadas, Universidade Federal do ABC, Santo André, 2016.

OLIVA, F.G. Relações entre o índice de oscilação antártica e as vazões na bacia hidrográfica do rio Uruguai, região Sul do Brasil. 2011. 68 f. Dissertação (Mestrado em Ciências) Instituto de Geociências, Universidade Federal do Rio de Janeiro, Rio de Janeiro, 2011.

OLIVEIRA, G.G.; PEDROLLO, O.C.; CASTRO, N.M. dos R. As incertezas associadas às condições climáticas obtidas pelo modelo ETA CPETC/HADCM3: Avaliação comparativa entre os dados simulados e observados de precipitação, evaporação e vazão na bacia hidrográfica do Rio Ijuí, Brasil. Revista Brasileira de Meteorologia, São Paulo, v. 30, n. 1, p. 101-121, 2015.

PICCILLI, D.G.A. Avaliação da precipitação hidroclimática no Alto Paraguai. 2007. 342 f. Tese (Doutorado em Recurso Hídrico e Saneamento Ambiental) - Instituto de Pesquisa Hidráulicas, Universidade Federal do Rio Grande do Sul, Porto Alegre, 2007.

PINTO, E.J.A.; NAGHETTINI, M.; ABREU, M.L., Utilização de indicadores climáticos na previsão probabilística de precipitações e vazões na Bacia do Alto São Francisco. Revista Brasileira de Recursos Hídricos, v. 11, n. 4, p. 121-135, 2006.

RATTON, P.; GUETTER, A.K. Previsão de vazões para o Rio Paraguai em Cáceres com atualização de estado. Parte II: Filtro de Kalman. In: Simpósio Brasileiro de Recursos Hídricos, 21., 2015, Brasília, Anais... Porto Alegre: ABRH, 2015.

ROBERTSON, A.W.; MECHOSO, C.R. Interannual and decadal cycles in river flows of southeastern South America.Journal of Climate, v. 11, n. 10, p. 2570-2581, 1998.

SIQUEIRA, A.H.B.; MOLION, L.C.B. Análises Climáticas: O filtro Hodrick-Prescott aplicado aos índices atmosféricos da Oscilação Sul e da Oscilação do Atlântico Norte. Revista Brasileira de Meteorologia, v. 30, n. 3, p. 307-318, 2015. 
STIVARI, S.M.S.; OLIVEIRA, A.P.; KARAM, H.A.; SOARES, J. Patters of local circulation in the Itaipu lake area: Numerical simulations of lake breeze. Journal of Applied Meteorology, n. 42, n. 1, p. 37-50, 2003.

SOUZA FILHO, F.A.; LALL, U. Modelo de previsão de vazões sazonais e interanuais. Revista Brasileira de Recursos Hídricos, v. 9, n. 2, p. 61-74, 2004.

TUCCI, C.E.M. Modelos Hidrológicos. 2 ed. Porto Alegre: UFRGS/ABRH, 2005, 680 p.
WAINER, I.; VENEGAS, S.A. South Atlantic multidecadal variability in the climate system model. Journal of Climate, v. 15, n. 12, p. 1408-1420, 2002.

WILKS, D.S. Statistical Methods in the Atmospheric Sciences. 2. ed. Philadelphia: Elsevier Academic Press Publications, 2006.

This is an Open Access article distributed under the terms of the Creative Commons Attribution Non-Commercial License which permits unrestricted non-commercial use, distribution, and reproduction in any medium provided the original work is properly cited. 